\title{
Nucleation and evolution of spherical crystals with allowance for their unsteady-state growth rates
}

\author{
D V Alexandrov \\ Department of Theoretical and Mathematical Physics, Laboratory of Multi-Scale \\ Mathematical Modeling, Ural Federal University, Lenin ave., 51, Ekaterinburg \\ 620000, Russia \\ E-mail: dmitri.alexandrov@urfu.ru
}

Received 23 December 2017

Accepted for publication 8 January 2018

Published 22 January 2018

\begin{abstract}
The growth dynamics of a spherical crystal in a metastable liquid is analyzed theoretically. The unsteady-state contributions to the crystal radius and its growth rate are found as explicit functions of metastability level $\Delta$ and time $t$. It is shown that the fundamental contribution to the growth rate represents the time independent solution of a similar temperature conductivity problem (Alexandrov and Malygin 2013 J. Phys. A: Math. Theor. 46 455101) whereas the next unsteady-state contribution is proportional to $\Delta^{2} t$. On the basis of these explicit unsteady-state solutions, the process of transient nucleation and growth of spherical crystals in a metastable system is theoretically studied at the intermediate stage of phase transformation. A complete analytical solution for the particle-radius distribution function and metastability level is constructed with allowance for the Weber-Volmer-Frenkel-Zel'dovich and Meirs kinetic mechanisms. It is shown that the obtained unsteady-state contribution to the crystal growth rate plays an important role in the nucleation process and drastically changes the particle-radius distribution function.
\end{abstract}

Keywords: phase transitions, nucleation, solidification

(Some figures may appear in colour only in the online journal)

\section{Introduction}

Evolution of particulate ensembles in a metastable liquid usually occurs as a result of nucleation and growth of individual particles whose coarsening reduces the level of system metastability (supercooling or supersaturation) and changes their size distribution function. A complete determination of unsteady-state behavior of the current metastability level and 
particle distribution density represents a challenging task of great importance in practice. Indeed, nucleation and crystal growth phenomena occur in different branches of applied science ranging from the crystallization and condensation processes in melts and solutions to life science, metallurgical and chemical industries such as manufacturing processes of many kinds of food, drug products and specialty chemicals [1-9].

Generally speaking, the phase transition process can be divided into three main stages, initial, intermediate and concluding, where various physical mechanisms of particle evolution predominate $[10,11]$. So, for example, crystals nucleate and grow under practically unchanged metastability level at the initial stage, they evolve and reduce this level at the intermediate stage whereas larger crystals grow at the expense of the dissolution of smaller ones at the concluding stage where coagulation and disintegration processes are possible as well [12-17]. The first and last stages were successively studied by a number of researches (see, among others, $[15,18-21])$. On the contrary, the intermediate stage is far from a complete analytical description due to the fact that its model represents a complex system of integrodifferential equations with moving boundaries of the phase transition. The effective methods of getting its reliable solutions have been detailed in [1, 2, 13, 22, 23] for molecular colloidal and polymerizing systems as well as for magnetic fluids. However, these methods are based on the stationary temperature or solute concentration fields around the growing spherical nuclei (see, for details, the growth rate of crystals found in [23] as the solution of time independent temperature conductivity equation). This approximation, strictly speaking, does not valid at all growth stages of a spherical crystallite releasing the latent heat of solidification, displacing solute impurities and changing the temperature (concentration) field near its evolving solid/ liquid boundary of the phase transition. The present paper shows that the growth of crystals in unsteady-state manner substantially changes the nonstationary process of evolution of a metastable system at the intermediate stage of the phase transition.

\section{Unsteady-state growth rate of spherical nuclei}

Let us consider the growth of spherical crystals in a metastable medium. We assume that the growing particles can interact only by means of the temperature field, which in turn they can change by means of the latent heat of phase transition. In addition, let us assume that the crystals are randomly distributed in the supercooled liquid and form the face-centered cubic lattice. Then the symmetry planes will divide the metastable volume into fine meshes, and the crystals evolve in their centers.

Let $R_{e}$ be the radius of equivalent sphere where the crystal of radius $R(t)$ grows with time $t$ (figure 1). Moreover, for the sake of simplicity, we neglect the critical radius of nucleating particles. The temperature field $T(r, t)$ in the liquid phase around the growing crystal satisfies the temperature conductivity equation

$$
\frac{\partial^{2} T}{\partial r^{2}}+\frac{2}{r} \frac{\partial T}{\partial r}=\frac{1}{a} \frac{\partial T}{\partial t}, R(t)<r<R_{e}
$$

where $r$ is the spherical coordinate and $a$ is the thermal diffusivity.

The boundary conditions at the phase interface (at $r=R(t)$ ) and at the equivalent sphere (at $r=R_{e}$ ) have the form

$$
\frac{\mathrm{d} R}{\mathrm{~d} t}=-\frac{\lambda_{l}}{\rho_{s} L} \frac{\partial T}{\partial r}=\beta_{*}\left(T_{p}-T\right), r=R(t) ; \frac{\partial T}{\partial r}=0, r=R_{e},
$$




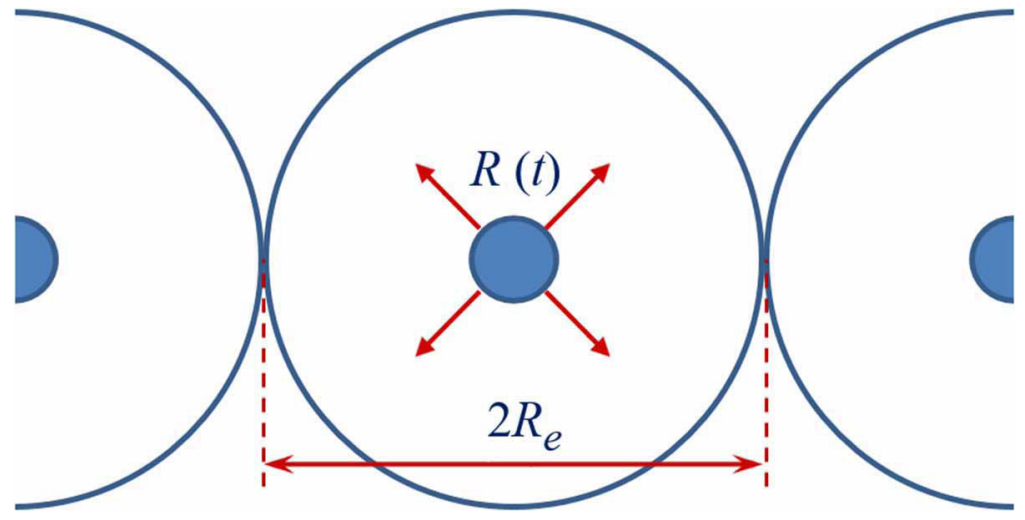

Figure 1. A scheme of crystal growth.

where $\lambda_{l}$ is the thermal conductivity, $\rho_{s}$ is the density of solid phase, $L$ is the latent heat of phase transition, $T_{p}$ is the phase transition temperature, and $\beta_{*}$ is the kinetic coefficient.

Let us introduce the following dimensionless variables

$$
\rho=\frac{r}{R_{e}}, \tau=\frac{a t}{R_{e}^{2}}, \rho_{0}(\tau)=\frac{R(t)}{R_{e}} .
$$

The problem (1), (2) in dimensionless variables (3) takes the form

$$
\begin{aligned}
& \frac{\partial^{2} T}{\partial \rho^{2}}+\frac{2}{\rho} \frac{\partial T}{\partial \rho}=\frac{\partial T}{\partial \tau}, \rho_{0}(\tau)<\rho<1, \\
& \frac{\mathrm{d} \rho_{0}}{\mathrm{~d} \tau}=-\Lambda \frac{\partial T}{\partial \rho}=\tilde{\beta}_{*}\left(T_{p}-T\right), \rho=\rho_{0}(\tau) ; \frac{\partial T}{\partial \rho}=0, \rho=1,
\end{aligned}
$$

where $\Lambda=\lambda_{l} /\left(\rho_{s} L a\right), \tilde{\beta}_{*}=R_{e} \beta_{*} / a$, and $\rho$ and $\tau$ play the role of dimensionless coordinate and time.

The solution of equation (4) satisfying the last boundary condition (5) can be written out as [24]

$$
T(\rho, \tau)=\frac{1}{\rho} \sum_{n=0}^{\infty} \frac{(1-\rho)^{2 n}}{(2 n) !}\left(1-\frac{1-\rho}{2 n+1}\right) \frac{\mathrm{d}^{n} B(\tau)}{\mathrm{d} \tau^{n}},
$$

where $B(\tau)=T(1, \tau)$.

Substitution of solution (6) into two boundary conditions (5) at $\rho=\rho_{0}$ leads to the following equations for the determination of $\rho_{0}(\tau)$ and $B(\tau)$

$$
\begin{aligned}
& \rho_{0} \frac{\mathrm{d} \rho_{0}}{\mathrm{~d} \tau}=\rho_{0} \beta(\tau)+\sum_{n=1}^{\infty} \frac{\left(1-\rho_{0}\right)^{2 n}}{(2 n) !}\left(1-\frac{1-\rho_{0}}{2 n+1}\right) \frac{\mathrm{d}^{n} \beta(\tau)}{\mathrm{d} \tau^{n}}, \\
& \left(P \rho_{0}+1\right) \frac{\mathrm{d} \rho_{0}}{\mathrm{~d} \tau}=\beta(\tau)-\sum_{n=1}^{\infty} \frac{\left(1-\rho_{0}\right)^{2 n-1}}{(2 n-1) !}\left(1-\frac{1-\rho_{0}}{2 n}\right) \frac{\mathrm{d}^{n} \beta(\tau)}{\mathrm{d} \tau^{n}},
\end{aligned}
$$

where $\beta(\tau)=\left[T_{p}-B(\tau)\right] \tilde{\beta}_{*}$ and $P=\tilde{\beta}_{*} / \Lambda$.

Designating $\beta_{0}=\beta(0)$ and estimating this parameter as $\beta_{0} \ll 1$ [23], we seek for the solution of system (7) and (8) in series in $\beta_{0}$ as 


$$
\rho_{0}(\tau)=\beta_{0} \varphi_{1}(\tau)+\beta_{0}^{2} \varphi_{2}(\tau)+\ldots, \beta(\tau)=\beta_{0} \psi_{1}(\tau)+\beta_{0}^{2} \psi_{2}(\tau)+\ldots
$$

Next substituting (9) into equations (7) and (8) and equating the coefficients at the same powers of $\beta_{0}$, we get

$$
\varphi_{1}=\tau, \psi_{1}=1, \varphi_{2}=-\frac{P \tau^{2}}{2}, \psi_{2}=0, \varphi_{j}(0)=0, \psi_{j}(0)=0, j \geqslant 2 .
$$

In order to find $\varphi_{3}(\tau)$ and $\psi_{3}(\tau)$, we have the following system

$$
\begin{aligned}
& -P \tau^{2}=\sum_{n=1}^{\infty}\left[\frac{1}{(2 n) !}-\frac{1}{(2 n+1) !}\right] \frac{\mathrm{d}^{n} \psi_{3}}{\mathrm{~d} \tau^{n}}, \\
& \frac{\mathrm{d} \varphi_{3}}{\mathrm{~d} \tau}-\frac{3}{2} P^{2} \tau^{2}=\psi_{3}-\sum_{n=1}^{\infty}\left[\frac{1}{(2 n-1) !}-\frac{1}{(2 n) !}\right] \frac{\mathrm{d}^{n} \psi_{3}}{\mathrm{~d} \tau^{n}} .
\end{aligned}
$$

Now applying the Laplace-Carson transform to equations (11) and (12) an taking into account that

$$
\begin{aligned}
& \sum_{n=1}^{\infty} \frac{s^{n}}{(2 n) !}=\cosh \sqrt{s}-1, \sum_{n=1}^{\infty} \frac{s^{n}}{(2 n+1) !}=\frac{1}{\sqrt{s}} \sinh \sqrt{s}-1, \\
& \sum_{n=1}^{\infty} \frac{s^{n}}{(2 n-1) !}=\sqrt{s} \sinh \sqrt{s},
\end{aligned}
$$

we obtain

$$
\begin{aligned}
& \varphi_{3}^{*}(s)=\frac{3 P^{2}}{s^{3}}+\frac{2 P(\sqrt{s} \sinh \sqrt{s}-\cosh \sqrt{s})}{s^{5 / 2}(\sqrt{s} \cosh \sqrt{s}-\sinh \sqrt{s})}, \\
& \psi_{3}^{*}(s)=-\frac{2 P}{s^{3 / 2}(\sqrt{s} \cosh \sqrt{s}-\sinh \sqrt{s})},
\end{aligned}
$$

where $s$ is the Laplace-Carson variable and superscript $*$ designates the Laplace-Carson transform.

In order to apply the inverse Laplace-Carson transform let us represent expressions in the right-hand sides of solutions (13) and (14) as

$$
\begin{aligned}
& \frac{\sqrt{s} \sinh \sqrt{s}-\cosh \sqrt{s}}{s^{5 / 2}(\sqrt{s} \cosh \sqrt{s}-\sinh \sqrt{s})}=-\frac{1}{s^{2}}+\frac{1}{s} \Phi_{1}^{*}(s) \Phi_{2}^{*}(s), \\
& \frac{1}{s^{3 / 2}(\sqrt{s} \cosh \sqrt{s}-\sinh \sqrt{s})}=\frac{1}{s} \Psi_{1}^{*}(s) \Psi_{2}^{*}(s),
\end{aligned}
$$

where

$$
\begin{aligned}
& \Phi_{1}^{*}(s)=\frac{1}{s}-\frac{1}{s^{2}}, \Phi_{2}^{*}(s)=\frac{\cosh \sqrt{s}}{\cosh \sqrt{s}-\frac{\sinh \sqrt{s}}{\sqrt{s}}}, \\
& \Psi_{1}^{*}(s)=\frac{1}{s^{2}}, \Psi_{2}^{*}(s)=\frac{s^{3 / 2}}{\sqrt{s} \cosh \sqrt{s}-\sinh \sqrt{s}} .
\end{aligned}
$$


Now taking into account that $[24,25]$

$$
\frac{1}{s^{n}} \rightarrow \frac{\tau^{n}}{n !}, \Phi_{2}^{*} \rightarrow \frac{6}{5}+2 \sum_{k=1}^{\infty} \frac{\exp \left(s_{k} \tau\right)}{s_{k}}, \Psi_{2}^{*} \rightarrow 3+2 \sum_{k=1}^{\infty} \frac{\sqrt{s_{k}} \exp \left(s_{k} \tau\right)}{\sinh \sqrt{s_{k}}}
$$

where $s_{k}$ represent the roots of transcendental equation

$$
\sqrt{s_{k}} \cosh \sqrt{s_{k}}-\sinh \sqrt{s_{k}}=0,
$$

we finally obtain from expressions (13) and (14) their originals

$$
\begin{aligned}
& \varphi_{3}(\tau)=\frac{P^{2} \tau^{3}}{2}+2 P\left[\frac{\tau^{2}}{10}-\frac{\tau^{3}}{5}-2 \sum_{k=1}^{\infty} \frac{\nu_{k}(\tau)}{\mu_{k}^{2}}\right] \\
& \psi_{3}(\tau)=2 P \sum_{k=1}^{\infty} \frac{2 \exp \left(-\mu_{k}^{2} \tau\right)+2 \mu_{k}^{2} \tau-\mu_{k}^{4} \tau^{2}-2}{\mu_{k}^{5} \sin \mu_{k}}-P \tau^{3},
\end{aligned}
$$

where $\sqrt{s_{k}}=\mathrm{i} \mu_{k}, \mu_{k}$ satisfy the characteristic equation $\mu_{k} \cos \mu_{k}=\sin \mu_{k}$, and

$$
\nu_{k}=\frac{1}{\mu_{k}^{4}}\left[\mu_{k}^{2} \tau-1+\exp \left(-\mu_{k}^{2} \tau\right)\right]-\frac{1}{\mu_{k}^{6}}\left[1+\frac{\mu_{k}^{4} \tau^{2}}{2}-\mu_{k}^{2} \tau-\exp \left(-\mu_{k}^{2} \tau\right)\right] .
$$

Substitution of $\varphi_{i}$ and $\psi_{i}$ from (10), (15) and (16) into (9) gives the dimensionless solutions for the crystal radius $\rho_{0}$ and reduced temperature $\beta$ in the form

$$
\rho_{0}(\tau)=\beta_{0} \tau-\frac{\beta_{0}^{2} P \tau^{2}}{2}+\beta_{0}^{3} \varphi_{3}(\tau), \beta(\tau)=\beta_{0}+\beta_{0}^{3} \psi_{3}(\tau)
$$

Here to determine the main contributions and their corrections for the crystal radius and the growth rate of particles, we neglected the terms proportional to $\beta_{0}^{4}$ (and also to the higher powers in $\beta_{0}$ ) considering the case $\beta_{0} \ll 1$.

Let us now express the main contributions that determine the growth dynamics of a spherical crystal in a metastable liquid. To do this, we rewrite the first expression (17) in dimensional form and restrict ourselves to the case of two main contributions. Thus, the crystal radius $R(t)$ and its growth rate $V(t)$ take the form

$$
R(t)=\beta_{*} \Delta t-\frac{\beta_{*}^{3} \Delta^{2} t^{2}}{2 a \Lambda}, V(t)=\frac{\mathrm{d} R}{\mathrm{~d} t}=\beta_{*} \Delta\left(1-\frac{\beta_{*}^{2} \Delta t}{a \Lambda}\right),
$$

where $\Delta=T_{p}-T(1,0)$. Moreover, at the intermediate stage of particle nucleation and growth when they evolve most independently of each other $T(1,0)$ represents the mean temperature of a metastable system $T_{l}$. It is significant to note that expressions (18) do not depend on $R_{e}$.

An important point is that the first contributions (proportional to $\Delta$ ) in expressions (18) describe the solution of the corresponding problem when the right-hand side of equation (1) is zero (time independent temperature conductivity equation) in kinetically-controlled growth regime [23]. Estimating $\beta_{*}^{2} \Delta t /(a \Lambda) \sim 10^{-2} t \mathrm{~s}^{-1}$ as a typical case for metallic melts, we conclude that the second term in expressions (18) becomes substantial at times $t \gtrsim 10 \mathrm{~s}$ after nucleation of a certain particle. In other words, the commonly used approximation of the particle growth rate $V(t) \approx \beta_{*} \Delta[13,14,22,26-29]$ is only the rough estimate that describes the main contribution only. In order to obtain a more precise description of the real nucleation process one can use next terms in the asymptotic expansion of $R(t)$ and $V(t)$ found in expressions (18). This conclusion is clearly demonstrated in the next section where we extend the 
theory of $[13,23]$ to the case of unsteady-state rate of spherical crystals in accordance with the second expression (18).

\section{Unsteady-state nucleation and growth of crystals}

Let us consider the evolution of crystals in a single-component metastable liquid (supercooled melt or supersaturated solution). The particle-radius density distribution function $f(r, t)$ satisfies the Fokker-Planck type kinetic equation (without diffusion term) whereas the system supercooling $\Delta(t)=T_{p}-T_{l}$ in the case of supercooled liquids (or the system supersaturation $\Delta(t)=C_{l}-C_{p}$ in the case of supersaturated solutions) is described by the heat (or mass) balance equation [30], i.e.

$$
\frac{\partial f}{\partial t}+\frac{\partial}{\partial r}(V(t) f)=0, \frac{\mathrm{d} \Delta}{\mathrm{d} t}=-a_{1} \int_{0}^{\infty} r^{2} V(t) f(r, t) \mathrm{d} r
$$

where $a_{1}=4 \pi \rho_{s} L /\left(\rho_{m} C_{m}\right)$ and $a_{1}=4 \pi C_{p}$ for supercooled melts (SM) and supersaturated solutions (SS), respectively. Here $\rho_{m}$ and $C_{m}$ are the density and specific heat of the melt, and $C_{l}$ and $C_{p}$ are the solute concentration and concentration at saturation.

The boundary conditions to equation (19) have the form

$$
f(r, 0)=0, \Delta(0)=\Delta_{0}, V(t) f(0, t)=I(\Delta),
$$

where $\Delta_{0}$ is the initial supercooling (supersaturation) of the metastable system, which previously did not contain any solid particles.

The nucleation rate $I(\Delta)$ in the case of the Weber-Volmer-Frenkel-Zel'dovich (WVFZ) and Meirs kinetics can be expressed as

$$
\begin{aligned}
& I_{\mathrm{WVFZ}}(\Delta)=I_{*} \exp \left(-\frac{p \Delta_{0}^{2}}{\Delta^{2}}\right) \text { for SM, } \\
& I_{\mathrm{WVFZ}}(\Delta)=I_{*} \exp \left(-\frac{p}{\ln ^{2}\left(C_{l} / C_{p}\right)}\right) \text { for SS, }
\end{aligned}
$$

and $I_{\text {Meirs }}(\Delta)=I_{*} \Delta^{p}$ for SM and SS systems. Here $I_{*}$ and $p$ entering in these expressions represent the different constants for the WVFZ and Meirs kinetics as well as for SM and SS systems (see, for details, [23, 30]). Note that the last boundary condition (20) describes the flux of nucleating crystals.

For the sake of convenience, let us introduce the following dimensionless variables and parameters

$$
\tau_{d}=\frac{t}{t_{0}}, z=\frac{r}{l_{0}}, w=\frac{\Delta}{\Delta_{0}}, F=l_{0}^{4} f, t_{0}=\frac{l_{0}}{\beta_{*} \Delta_{0}}, l_{0}=\left(\frac{\beta_{*} \Delta_{0}}{I_{0}}\right)^{1 / 4},
$$

where $I_{0}=I\left(\Delta_{0}\right)$ and $\Delta_{0}$ stands for the initial value of system metastability $\Delta$.

Rewriting equation (19) and boundary conditions (20) in dimensionless variables (21), we come to

$$
\begin{aligned}
& \frac{\partial F}{\partial \tau_{d}}+V_{d}\left(\tau_{d}\right) \frac{\partial F}{\partial z}=0, \frac{\mathrm{d} w}{\mathrm{~d} \tau_{d}}=-b_{1} V_{d}\left(\tau_{d}\right) \int_{0}^{\infty} z^{2} F\left(z, \tau_{d}\right) \mathrm{d} z \\
& F(z, 0)=0, w(0)=1, F\left(0, \tau_{d}\right)=\frac{\exp (p g(w))}{V_{d}\left(\tau_{d}\right)}
\end{aligned}
$$


where

$$
g(w)=g_{\mathrm{WVFZ}}(w)=\left\{\begin{array}{l}
1-w^{-2}, \mathrm{SM} \\
\ln ^{-2}\left(1+w_{p}^{-1}\right)-\ln ^{-2}\left(1+w / w_{p}\right), \mathrm{SS}
\end{array}\right.
$$

and $g(w)=g_{\text {Meirs }}(w)=\ln w$ for the supercooled and supersaturated systems $[10,11,30]$. Here the following designations are introduced: $b_{1}=a_{1} / \Delta_{0}, V_{d}\left(\tau_{d}\right)=V t_{0} / l_{0}=w\left(\tau_{d}\right)-\sigma w^{2}\left(\tau_{d}\right) \tau_{d}$, and $\sigma=\beta_{*} l_{0} /(a \Lambda)$, and $z$ and $\tau_{d}$ represent the dimensionless coordinate and time.

The exact solution of the first equation (22) supplemented by the initial and boundary conditions (23) has the form

$F(x, z)=\phi\left(x\left(\tau_{d}\right)-z\right), \phi=\frac{\exp \left[p g\left(w\left(\tau_{d}\right)\right)\right]}{V_{d}\left(\tau_{d}\right)}, x\left(\tau_{d}\right)=\int_{0}^{\tau_{d}} V_{d}\left(\tau_{1}\right) \mathrm{d} \tau_{1}$.

Let us now introduce the new variable $x(\nu)=x\left(\tau_{d}\right)-z$ so that at $\nu=0$ the maximum radius of crystals is $z_{m}=x\left(\tau_{d}\right)$ and the limits $z=0$ and $z=z_{m}$ of integration with respect to $z$ transform to the corresponding limits $\nu=\tau_{d}$ and $\nu=0$ of integration with respect to variable $\nu$. Taking this into account, we rewrite the second equation (22) as

$\frac{\mathrm{d} w}{\mathrm{~d} \tau_{d}}=-b_{1} V_{d}\left(\tau_{d}\right) \int_{0}^{\tau_{d}} h\left(\nu, \tau_{d}\right) \exp (p g(\nu)) \mathrm{d} \nu, h\left(\nu, \tau_{d}\right)=\left[x\left(\tau_{d}\right)-x(\nu)\right]^{2}$.

A moment's consideration shows that $\mathrm{d} g / \mathrm{d} \nu<0$ for the WVFZ and Meirs nucleation kinetics. So, for instance, in the case of supercooled liquids one can get $\mathrm{d} g / \mathrm{d} \nu=2 w^{-3} \mathrm{~d} w / \mathrm{d} \nu$ (WVFZ) and $\mathrm{d} g / \mathrm{d} \nu=w^{-1} \mathrm{~d} w / \mathrm{d} \nu$ (Meirs), where $w(\nu)$ is a monotonically decreasing function as a result of particle growth process. Keeping this in mind, we conclude that $g(\nu)$ takes its maximum at $\nu=0$. In order to apply the saddle-point method for the Laplace-type integral (25) [31], we find the first nonzero derivative at $\nu=0$ using the same equation (25). One can easily show that the first three derivatives at $\nu=0$ vanish whereas $g^{(\mathrm{IV})}=-4 b_{1}$ for the WVFZ and $g^{(\mathrm{IV})}=-2 b_{1}$ for the Meirs kinetics at $\nu=0$. Taking all of the above into account and keeping in mind the fundamental contribution of the saddle-point method, we have [31]

$$
\frac{\mathrm{d} w}{\mathrm{~d} x}=-\Sigma x^{2}, \Sigma=\frac{b_{1}}{4 p^{1 / 4}} \Gamma\left(\frac{1}{4}\right)\left(-\frac{24}{g^{(\mathrm{IV})}}\right)^{1 / 4},
$$

where the initial condition becomes $w=1$ at $x=0$. Integration of equation (26) gives the dimensionless metastability level

$$
w(x)=1-\frac{\Sigma x^{3}}{3}
$$

and the last expression (24) determines the Cauchy problem for $\tau_{d}(x)$

$$
\frac{\mathrm{d} \tau_{d}}{\mathrm{~d} x}=\frac{1}{w(x)\left[1-\sigma w(x) \tau_{d}(x)\right]}, \tau_{d}=0 \text { at } x=0 .
$$

Now the first two expressions (24) lead to the dimensionless distribution function of the form

$$
F(x, z)=\frac{\exp [p g(w(x-z))]}{w(x-z)\left[1-\sigma \tau_{d}(x-z) w(x-z)\right]} .
$$

Note that in expressions (27)-(29), $w, \tau_{d}, x$ and $z$ represent the dimensionless supercooling (supersaturation), time, modified time and spatial coordinate, respectively. 


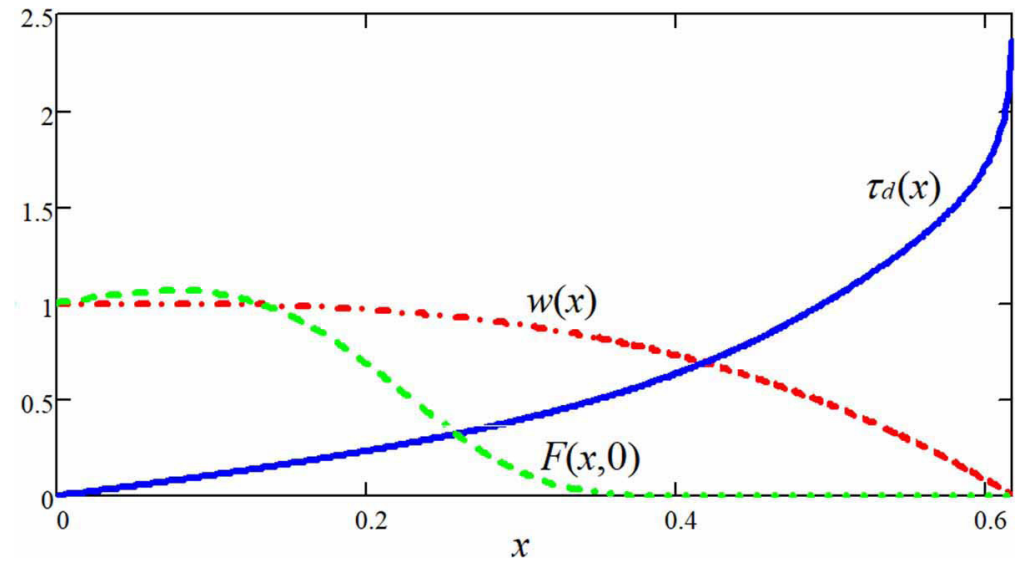

Figure 2. Parametric solutions (27)-(29); physical parameters used for calculations are [23]: $\rho_{s}=7.8 \cdot 10^{3} \mathrm{~kg} \mathrm{~m}^{-3}, \rho_{m}=7 \cdot 10^{3} \mathrm{~kg} \mathrm{~m}^{-3}, L=9 \cdot 10^{5} \mathrm{~J} \mathrm{~kg}^{-1}, C_{m}=840$ $\mathrm{J} \mathrm{kg}^{-1} \mathrm{~K}^{-1}, \Delta_{0}=373 \mathrm{~K}, p=10, \beta_{*}=8 \cdot 10^{-6} \mathrm{~m} \mathrm{~s}^{-1} \mathrm{~K}^{-1}, I_{0}=10^{9} \mathrm{~m}^{-3} \mathrm{~s}^{-1}, \lambda_{l}=63$ $\mathrm{J} \mathrm{m}^{-1} \mathrm{~K}^{-1} \mathrm{~s}^{-1}, \Lambda a=9 \cdot 10^{-9} \mathrm{~m}^{2} \mathrm{~K}^{-1} \mathrm{~s}^{-1}$.

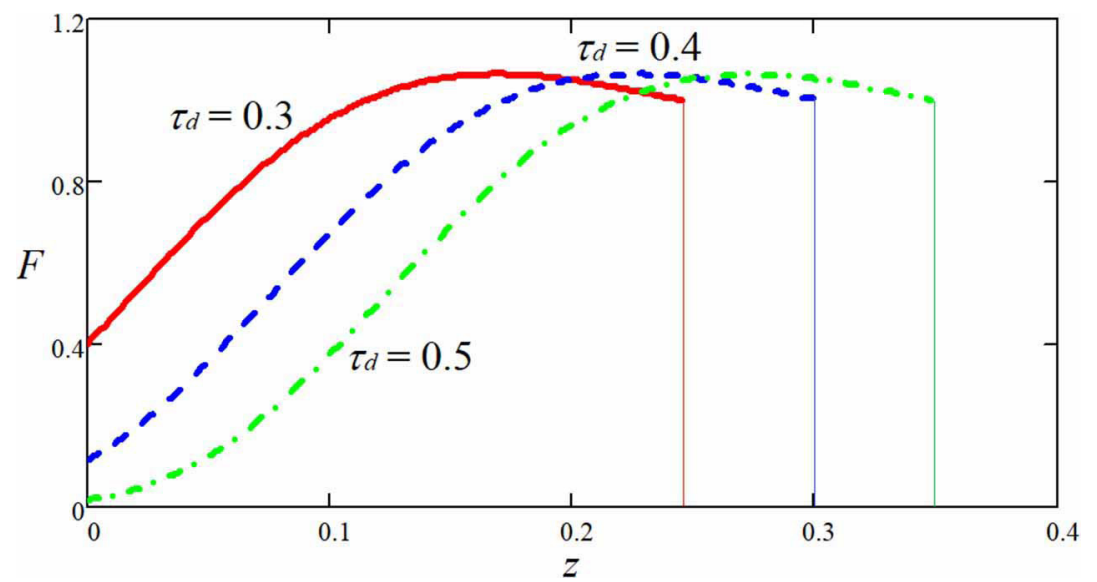

Figure 3. Dimensionless distribution function versus dimensionless radius at different times. The vertical lines illustrate the maximum size $z_{m}$ of crystals.

Thus, expressions (27)-(29) represent a complete analytical solution of the nucleation problem in a parametric form (with parameter $x$ ) with allowance for the unsteady-state growth rate (18) of spherical crystals in a supercooled or supersaturated liquids. An important point is that the obtained solutions transform to the previously known solutions ([23]) following from the time independent temperature conductivity equation (when $V=\beta_{*} \Delta$ ).

It is important to keep in mind that the obtained solutions enable us to express the explicit expressions for the system supercooling (supersaturation) and crystal radius. Eliminating variable $x$ from expression (28) by means of formula (27) and taking into account variables (21), we come to the following explicit solutions

$$
\frac{\mathrm{d} w}{\mathrm{~d} t}=\zeta_{1} w(1-w)^{2 / 3}\left(1-\zeta_{2} t w\right), w=1, t=0,
$$




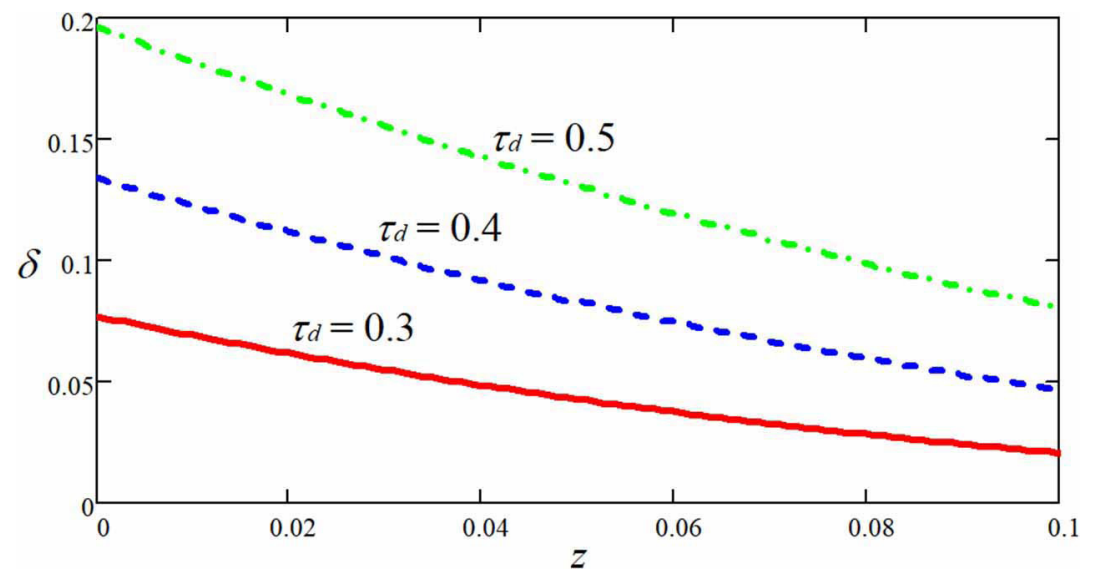

Figure 4. Relative error $\delta=\left[F\left(x\left(\tau_{d}\right), z\right)-F^{0}\left(x\left(\tau_{d}\right), z\right)\right] / F\left(x\left(\tau_{d}\right), z\right)$ of the distribution function (29), which takes into account the unsteady-state growth velocities of nuclei, relative to the distribution function $F^{0}\left(x\left(\tau_{d}\right), z\right)$, which takes into account only the main contribution $V=\beta_{*} \Delta$ (see, for details, [23]).

$$
\Delta(t)=\Delta_{0} w(t), R(t)=\beta_{*} \Delta(t) t-\frac{\beta_{*}^{3}(\Delta(t))^{2} t^{2}}{2 a \Lambda},
$$

where $\zeta_{1}=-3^{2 / 3} \Sigma^{1 / 3} / t_{0}$ and $\zeta_{2}=\sigma / t_{0}$. Note that the dimensionless metastability level as a function of time, $w(t)$, is determined from the Cauchy problem (30), whereas the time evolution of dimensional supercooling (supersaturation) and crystal radius is given by expressions (31) after the substitution of solution for $w(t)$

\section{Concluding remarks}

A complete parametric solution (27)-(29) is illustrated in figure 2. As would be expected, the metastability level $w(x)$ decreases with increasing the dimensionless time $x$ (or $\tau_{d}$ ). This is related to the fact that the growing crystals release the latent heat of solidification and thus reduce the supercooling of metastable medium. The particle-radius distribution function is shown in figure 3 at different times. In the absence of 'diffusion' term in the Fokker-Planck equation (19) [16], this function is bounded by the maximum radius $z_{m}$ of crystals that is available in the system at a given time. It is significant that the nonstationary contribution to the growth rate of spherical crystals (the second term in expression (18) for $V(t)$ ) plays a fundamental role in the evolutionary behavior of growing particles. Indeed, figure 4 demonstrates the relative error for the distribution function calculated as a relative difference in $F$ in the presence and absence of the nonstationary contribution in (18) for $V(t)$, which is proportional to $\Delta^{2} t$. As is easy to see, the role of this contribution increases with time and its magnitude may be as high as tens of percents. For this reason, the unsteady-state contributions in expressions for $R(t)$ and $V(t)$ (the last terms in expressions (18)) have to be taken into account when considering the processes of nucleation and growth of spherical crystals (phase separation in colloids [1], evolution of droplike aggregates in magnetic fluids [2], combined polymerization and crystallization [22], solidification of terrestrial magma oceans and lava lakes [32, 33], solidification with mushy and slurry layers [34-37] may be mentioned in this connection). 


\section{Acknowledgments}

This work was supported by the Russian Foundation for Basic Research (project no 16-08-00932).

\section{Appendix. Next time correction to the growing radius of spherical crystals}

Let us now calculate the next correction to the first expression (18). To do this, we rewrite the first expression (17) for the dimensionless radius of growing crystals in dimensional form and use formulas (3) and (15). As a result, we obtain

$R(t)=\beta_{*} \Delta t-\frac{\beta_{*}^{3} \Delta^{2} t^{2}}{2 a \Lambda}+\frac{\beta_{*}^{5} \Delta^{3} t^{3}}{2 a^{2} \Lambda^{2}}+\frac{2 \beta_{*}^{4} \Delta^{3} R_{e}^{5}}{a^{4} \Lambda}\left[\frac{a^{2} t^{2}}{10 R_{e}^{4}}-\frac{a^{3} t^{3}}{5 R_{e}^{6}}-2 \sum_{k=1}^{\infty} \frac{\nu_{k}(t)}{\mu_{k}^{2}}\right]$,

where $\mu_{k}$ and $\nu_{k}(t)$ are determined by the following expressions

$\mu_{k} \cos \mu_{k}=\sin \mu_{k}, \nu_{k}(t)=\frac{1}{\mu_{k}^{2}}\left(1+\frac{1}{\mu_{k}^{2}}\right)\left\{\frac{a t}{R_{e}^{2}}+\frac{1}{\mu_{k}^{2}}\left[\exp \left(-\frac{\mu_{k}^{2} a t}{R_{e}^{2}}\right)-1\right]\right\}-\frac{a^{2} t^{2}}{2 \mu_{k}^{2} R_{e}^{4}}$.

Note that the growth rate of crystals $V(t)=\mathrm{d} R / \mathrm{d} t$ should be substituted into the kinetic and balance equations for more precise modeling of the nucleation and evolution of particles at the intermediate stage of phase transformation process.

\section{ORCID iDs}

D V Alexandrov $\odot$ https://orcid.org/0000-0002-6628-745X

\section{References}

[1] Buyevich Y A and Ivanov A O 1993 Kinetics of phase separation in colloids: II. Non-linear evolution of a metastable colloid Physica A $193221-40$

[2] Ivanov A O and Zubarev A Y 1998 Non-linear evolution of a system of elongated droplike aggregates in a metastable magnetic fluid Physica A 251 348-67

[3] Galkin O and Vekilov P G 2000 Are nucleation kinetics of protein crystals similar to those of liquid droplets? J. Am. Chem. Soc. 122 156-63

[4] Hamza M A, Berge B, Mikosch W and Rühl E 2004 Homogeneous nucleation of supersaturated KCl-solutions from single levitated microdroplets Phys. Chem. Chem. Phys. 6 3484-9

[5] Barlow D A 2009 Theory of the intermediate stage of crystal growth with applications to protein crystallization J. Cryst. Growth 311 2480-3

[6] Kelton K F and Greer A L 2010 Nucleation in Condensed Matter: Applications in Materials and Biology (Amsterdam: Elsevier)

[7] Hanhoun M, Montastruc L, Azzaro-Pntel C, Biscans B, Freche M and Pibouleau L 2013 Simultaneous determination of nucleation and crystal growth kinetics of struvite using a thermodynamic modeling approach Chem. Eng. J. 215-6 903-12

[8] Diemand J, Angélil R, Tanaka K K and Tanaka H 2013 Large scale molecular dynamics simulations of homogeneous nucleation J. Chem. Phys. 139074309

[9] Angélil R, Diemand J, Tanaka K K and Tanaka H 2015 Homogeneous SPC/E water nucleation in large molecular dynamics simulations J. Chem. Phys. 143064507

[10] Alexandrov D V and Nizovtseva I G 2014 Nucleation and particle growth with fluctuating rates at the intermediate stage of phase transitions in metastable systems Proc. R. Soc. A 47020130647 
[11] Alexandrov D V 2014 On the theory of transient nucleation at the intermediate stage of phase transitions Phys. Lett. A 378 1501-4

[12] Slezov V V and Sagalovich V V 1987 Diffusive decomposition of solid solutions Sov. Phys.—Usp. $3023-45$

[13] Buyevich Y A and Mansurov V V 1990 Kinetics of the intermediate stage of phase transition in batch crystallization J. Cryst. Growth 104 861-7

[14] Buyevich Y A, Mansurov V V and Natalukha I A 1991 Instability and unsteady processes of the bulk continuous crystallization-I. Linear stability analysis Chem. Eng. Sci. 46 2573-8

[15] Slezov V V 2009 Kinetics of First-Order Phase Transitions (Weinheim: Wiley)

[16] Alexandrov D V and Malygin A P 2014 Nucleation kinetics and crystal growth with fluctuating rates at the intermediate stage of phase transitions Modelling Simul. Mater. Sci. Eng. 22015003

[17] Alexandrov D V 2014 Nucleation and crystal growth in binary systems J. Phys. A: Math. Theor. 47125102

[18] Zettlemoyer A C 1969 Nucleation (New York: Decker)

[19] Lifshitz I M and Slyozov V V 1961 The kinetics of precipitation from supersaturated solid solutions J. Phys. Chem. Solids 19 35-50

[20] Slezov V V 1978 Formation of the universal distribution function in the dimension space for newphase particles in the diffusive decomposition of the supersaturated solid solutions $J$. Phys. Chem. Solids 39 367-74

[21] Alexandrov D V 2015 On the theory of Ostwald ripening: formation of the universal distribution J. Phys. A: Math. Theor. 48035103

[22] Buyevich Y A and Natalukha I A 1994 Unsteady processes of combined polymerization and crystallization in continuous apparatuses Chem. Eng. Sci. 49 3241-7

[23] Alexandrov D V and Malygin A P 2013 Transient nucleation kinetics of crystal growth at the intermediate stage of bulk phase transitions J. Phys. A: Math. Theor. 46455101

[24] Lubov B Y 1975 The Theory of Crystallization in Large Volumes (Moscow: Nauka)

[25] Ditkin V A and Prudnikov A P 1965 Integral Transforms and Operational Calculus (New York: Pergamon)

[26] Mullin J W 1972 Crystallization (London: Butterworths)

[27] Kirkaldy J S and Young D J 1987 Diffusion in Condensed State (London: Institute of Metals)

[28] Mansurov V V 1990 The nonlinear dynamics of solidification of a binary melt with a nonequilibrium mushy region Math. Comput. Modelling 14 819-21

[29] Barlow D A, Baird J K and Su C-H 2004 Theory of the von Weimarn rules governing the average size of crystals precipitated from a supersaturated solution J. Cryst. Growth 264 417-23

[30] Alexandrov D V 2014 Nucleation and crystal growth kinetics during solidification: the role of crystalline withdrawal rate and external heat and mass sources Chem. Eng. Sci. 117 156-60

[31] Fedoruk M V 1977 Saddle-Point Method (Moscow: Nauka)

[32] Solomatov V S and Stevenson D J 1993 Kinetics of crystal growth in a terrestrial magma ocean J. Geophys. Res. 98 5407-18

[33] Alexandrov D V, Netreba A V and Malygin A P 2012 Time-dependent crystallization in magma chambers and lava lakes cooled from above: The role of convection and kinetics on nonlinear dynamics of binary systems Int. J. Heat Mass Trans. 55 1189-96

[34] Martin S and Kauffman P 1974 The evolution of under-ice melt ponds, or double diffusion at the freezing point J. Fluid Mech. 64 507-27

[35] Alexandrov D V and Ivanov A O 2000 Dynamic stability analysis of the solidification of binary melts in the presence of a mushy region: changeover of instability J. Cryst. Growth $210797-810$

[36] Herlach D M 2008 Phase Transformations in Multicomponent Melts (Weinheim: Wiley)

[37] Alexandrov D V and Malygin A P 2012 Flow-induced morphological instability and solidification with the slurry and mushy layers in the presence of convection Int. J. Heat Mass Trans. 55 3196-204 